\title{
High-Brilliance Ultranarrow-Band X Rays via Electron Radiation in Colliding Laser Pulses
}

\author{
Q.Z. Lv®, ${ }^{1, *}$ E. Raicher, ${ }^{2, \dagger}$ C. H. Keitel ${ }^{1}{ }^{1}$ and K. Z. Hatsagortsyan $\odot^{1}$ \\ ${ }^{1}$ Max-Planck-Institut für Kernphysik, Saupfercheckweg 1, 69117 Heidelberg, Germany \\ ${ }^{2}$ Soreq Nuclear Research Center, 81800 Yavne, Israel
}

(Received 28 June 2021; revised 8 December 2021; accepted 17 December 2021; published 13 January 2022)

\begin{abstract}
A setup of a unique $\mathrm{x}$-ray source is put forward employing a relativistic electron beam interacting with two counterpropagating laser pulses in the nonlinear few-photon regime. In contrast to Compton scattering sources, the envisaged x-ray source exhibits an extremely narrow relative bandwidth of the order of $10^{-4}$, comparable with an x-ray free-electron laser. The brilliance of the $\mathrm{x}$ rays can be an order of magnitude higher than that of a state-of-the-art Compton source. By tuning the laser intensities and the electron energy, one can realize either a single peak or a comblike x-ray source of around keV energy. The laser intensity and the electron energy in the suggested setup are rather moderate, rendering this scheme compact and tabletop size, as opposed to x-ray free-electron laser and synchrotron infrastructures.
\end{abstract}

DOI: 10.1103/PhysRevLett.128.024801

Ever since the discovery by W. C. Röntgen in 1895 , powerful x-ray techniques have been developed for determining the structure of matter at the atomic length scale $[1,2]$. Remarkable advancements have been achieved with the employment of synchrotron radiation [3-6] and the x-ray free-electron laser [7-12], which dramatically increased the brightness of the source. Unfortunately, the large size and cost of these facilities limit their accessibility to a wide community. Alternative schemes rely on Thomson and Compton scattering (CS) [13-16] and recently also on the radiation from laser-plasma interactions [17-20]. The advancement of compact and powerful laser systems revived interest in these sources [21-36]. The CS source is based on a collision of a laser pulse with a relativistic electron beam [Fig. 1(a)]. While not competing with the brightness of large facilities, CS sources have several advantages, providing $\mathrm{x}$-ray photons at a tunable energy in a broad spectral range and being relatively compact and affordable.

A compact brilliant $\mathrm{x}$-ray light source with narrow bandwidth $(\mathrm{BW})$ is an attractive tool, e.g., for $\mathrm{x}$-ray imaging in biology [37], x-ray nanoscale diagnostics in material science [38-40], and x-ray spectroscopy of highly charged ions [41,42]. Recently, a new field of x-ray quantum optics has been advanced aimed at the coherent

Published by the American Physical Society under the terms of the Creative Commons Attribution 4.0 International license. Further distribution of this work must maintain attribution to the author(s) and the published article's title, journal citation, and DOI. Open access publication funded by the Max Planck Society. control of atomic nuclei using shaped resonant $\mathrm{x}$ rays [43-49] that requires especially narrow BW x-ray beams. Different schemes for narrowing the $\mathrm{x}$-ray $\mathrm{BW}$ have been proposed that involve temporal laser pulse chirping [28-33] or temporally varying polarization [34] to compensate the nonlinear spectrum broadening. Alternatively, the x-ray photon yield at low BW can be enhanced using a travelingwave setup that allows an overlap of electron and laser beams longer than the Rayleigh length $[35,36]$. However, all these approaches require the precise control of the pulse shape, phase, or polarization, which is difficult in the high intensity domain.

In this Letter, an alternative approach for narrow BW bright $\mathrm{x}$ rays is put forward. Rather than modifying the laser pulse, an additional laser beam copropagating with the electrons is introduced. Namely, the setup consists of a relativistic electron beam interacting with two counterpropagating waves (CPW) [Fig. 1(b)]. The electron motion features two typical frequencies separated by orders of magnitude because of the Doppler effect, $\omega_{1}=\omega_{0}(1+$ $\left.v_{z}\right) \approx 2 \omega_{0}$ and $\omega_{2}=\omega_{0}\left(1-v_{z}\right) \approx \omega_{0} / 2 \gamma_{*}^{2}$, where $\omega_{0}$ is the laser frequency and $v_{z}$ the relativistic average velocity on axis and $\gamma_{*}$ the effective Lorentz factor [50] (units $\hbar=c=1$ are used throughout). Because of the nonlinearity of the relativistic dynamics, the electron absorbs several photons in both frequencies in the considered regime when emitting an x-ray photon. As a result, in the emission spectrum the Doppler-shifted high frequency $\omega_{1}$ peak is accompanied with satellites of $\omega_{0}$ separation. While the gross features of the spectrum (the spectral envelope) are determined by the counterpropagating laser beam, the subtle features are governed by the second copropagating laser beam. Accordingly, the $\mathrm{BW}$ of 


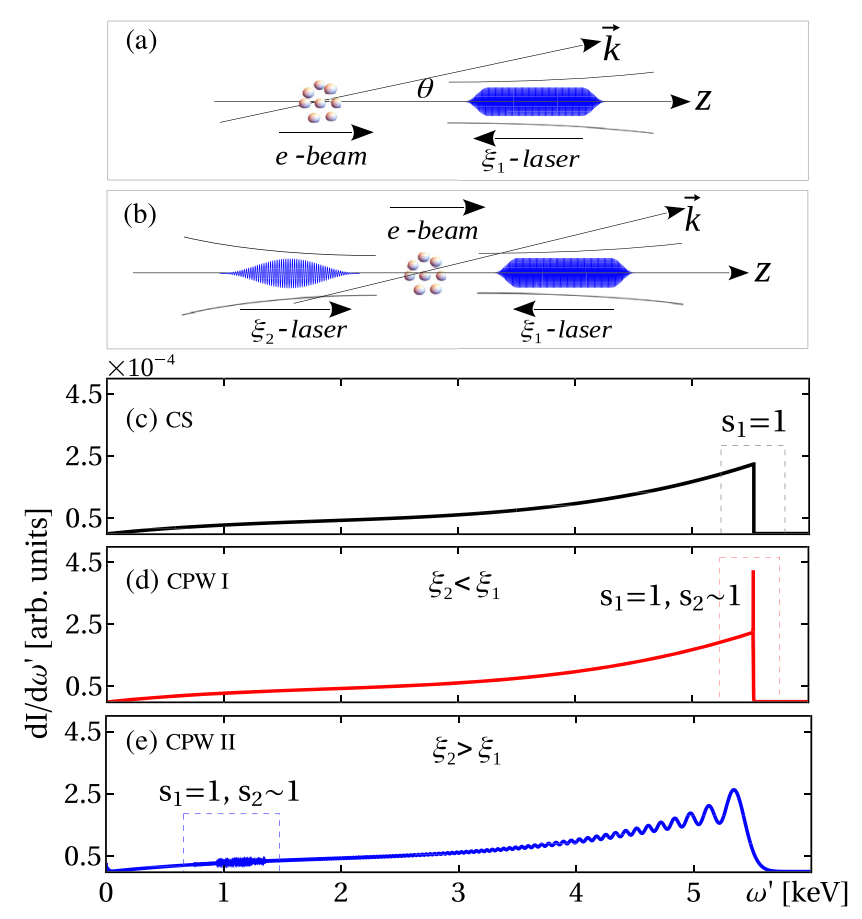

FIG. 1. Setup of a relativistic electron beam colliding with a single laser pulse for CS (a) and with CPW (b). The emission spectra for CS (c), for Case I (d), and Case II (e). The electron energy is $\varepsilon=30 \mathrm{~m}$ in all cases. $s_{i}$ denotes the absorbed photon numbers from the laser $\xi_{i}(i=1,2)$, respectively.

satellites scales with the smaller frequency $\omega_{2}$, allowing for bright ultranarrow $\mathrm{BW} x$ rays.

The radiation has been calculated employing the BaierKatkov semiclassical operator method [51], applicable when the electron dynamics is quasiclassical in strong background fields. The radiation spectrum reads [52,53]

$$
d I=\frac{\alpha}{(2 \pi)^{2} \tau}\left[-\frac{\varepsilon^{\prime 2}+\varepsilon^{2}}{2 \varepsilon^{\prime 2}}\left|\mathcal{T}_{\mu}\right|^{2}+\frac{m^{2} \omega^{\prime 2}}{2 \varepsilon^{\prime 2} \varepsilon^{2}}|\mathcal{I}|^{2}\right] d^{3} \boldsymbol{k}^{\prime}
$$

where $\mathcal{I} \equiv \int_{-\infty}^{\infty} e^{i \psi} \mathrm{d} t, \mathcal{T}_{\mu} \equiv \int_{-\infty}^{\infty} v_{\mu}(t) e^{i \psi} \mathrm{d} t, \psi \equiv\left(\varepsilon / \varepsilon^{\prime}\right) k^{\prime}$. $x(t)$ is the emission phase, and $x_{\mu}(t), v_{\mu}(t), k_{\mu}^{\prime}=\left(\omega^{\prime}, \boldsymbol{k}^{\prime}\right)$ are the four-vectors of the electron coordinate, the velocity, and the emitted photon momentum, respectively. $\tau$ is the pulse duration, $\varepsilon$ the electron energy with $\varepsilon^{\prime}=\varepsilon-\omega^{\prime}$. In our setup, ultrarelativistic electrons counterpropagate to the circularly polarized laser field with a vector potential $A_{1}(x, t)=m \xi_{1}\left[\cos \left(k_{1} \cdot x\right) e_{x}+\sin \left(k_{1} \cdot x\right) e_{y}\right]$, where $\xi_{1}=e E_{0} /\left(m \omega_{0}\right), E_{0}$ and $\omega_{0}$ are the laser field amplitude and frequency, respectively. $k_{1}=\left(\omega_{0}, 0,0,-\omega_{0}\right)$ is the laser four-wave vector with $\omega_{0}=1.55 \mathrm{eV}$, and $e_{x}=(0,1,0,0)$, $e_{y}=(0,0,1,0) .-e$ and $m$ are the electron charge and mass, respectively. The second laser field copropagating with the electrons is also circularly polarized: $A_{2}(x, t)=$ $m \xi_{2}\left[\cos \left(k_{2} \cdot x\right) e_{x}+\sin \left(k_{2} \cdot x\right) e_{y}\right]$, with $k_{2}=\left(\omega_{0}, 0,0, \omega_{0}\right)$. The two lasers have the same frequency $\omega_{0}$ in the lab frame. We keep $\xi_{1}<1$, namely $\xi_{1}=0.1$, while choosing either $\xi_{2}<\xi_{1}$ (Case I, $\xi_{2}=0.02$ ) or $\xi_{2}>\xi_{1}$ (Case II, $\xi_{2}=2$ ). The quantum strong-field parameter $\chi=e \sqrt{-\left(F^{\mu \nu} P_{\nu}\right)^{2}} / m^{3}$, with the field tensor $F^{\mu \nu}$, and four-momentum $P_{\nu}$, is small- $\chi \sim 10^{-5}$ for the chosen parameters-and multiple photon emissions are negligible.

The radiation spectra are presented in Fig. 1. The general features of all the spectra are similar. In the CS case, $\xi_{2}=0$ [Fig. 1(c)], the spectrum has a sharp edge corresponding to absorption of a single photon (as $\xi_{1}<1$ ) from the laser field. The same edge is also dominant in spectra for Cases I and II in CPW because $\chi$, determining the general spectral shape, is dominated by the $\xi_{1}$ laser. However, the details of the spectra in CPW reveal features stemming from the $\xi_{2}$ laser that are absent in CS. For Case I [Fig. 1(d)], a single but ultranarrow harmonic rises with a similar location but larger strength near the spectrum edge. For Case II [Fig. 1(e)], the entire spectrum becomes oscillatory and in most of the energy domain these oscillations are quite wide. However, the radiation emitted on axis, corresponding to $s_{2} \sim 1$, exhibits a comb of sharp peaks, which is the main interest here and discussed below.

The angle-resolved spectra are presented in Fig. 2 for CS and CPW Case I and Fig. 3 for CPW Case II. While in the CS case a single harmonic appears in the given limited spectral range [Fig. 2(a)], in CPW many satellites accompany the given harmonic due to absorption of additional photons from the $\xi_{2}$ laser [Figs. 2(d) and 3(a)]. Generally, the radiation is distributed in a rather large angle and frequency region. Since $\xi_{1}<1$, the photon number for both CS and Case I in CPW decreases monotonically in the $1 / \gamma$ cone, while the BW increases with $\theta$ until it reaches a constant value [50]. This makes the brilliance for both cases being the largest in the forward direction. Moreover, by integrating over the same angle range around $\theta=0$, we can see that the radiation in Case I is narrower in $\mathrm{BW}$ and more intense compared with CS [Figs. 2(b),(e)], which finally results in higher brilliance. After taking into account that the energy spreading and emittance of a realistic electron beam broaden the emission BW [Figs. 2(g), (h)], the advantage of Case I in brilliance compared to CS still remains at about 1 order of magnitude.

Furthermore, a comblike structure is produced in Case II [Figs. 3(b),(c)]. While state-of-the-art techniques for a frequency comb can achieve the extreme ultraviolet domain [54-57], the covet is the hard x-ray regime. An attempt in this direction recently shown in [34] via CS with a polarization gating demonstrated a relative $\mathrm{BW}$ of $10^{-2}$ and the spacing between peaks of the order of $\sim 100 \mathrm{keV}$. In our CPW setup, however, the comb spacing is at an optical frequency, with more than 1 order of magnitude smaller relative BW [Fig. 3(d)].

These results raise several questions: (i) What determines the peak locations and the spacing between sequential peaks? (ii) What determines the width of a single peak? (iii) What is the role played by the angle window? (iv) How 


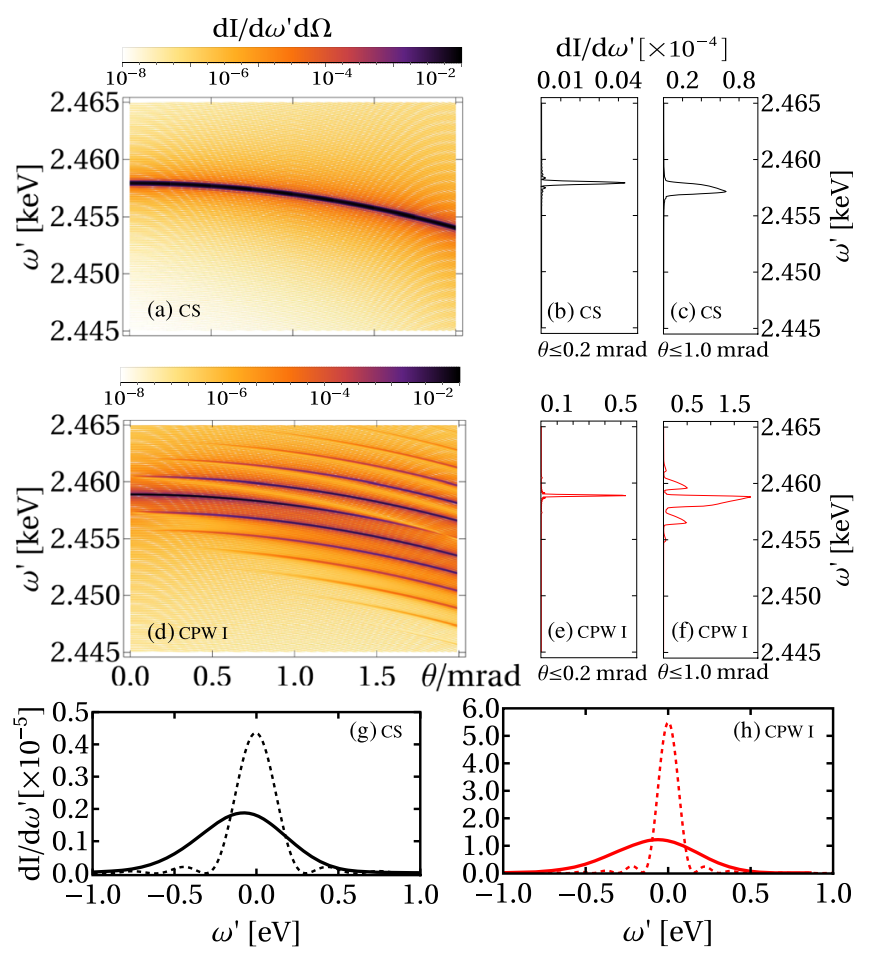

FIG. 2. The angle-resolved spectra: (a) CS; (d) CPW Case I. The angle-integrated spectra: within $\theta \leq 0.2 \mathrm{mrad}$ for (b) CS; (e) CPW Case I and within $\theta \leq 1 \mathrm{mrad}$ for (c) CS; (f) CPW Case I. The spectra from a realistic electron beam (solid) and a monochromatic electron beam (dashed): (g) for CS; (h) for CPW Case I. The $x$ axis in panels $(\mathrm{g}),(\mathrm{h})$ has been shifted by the peak energy in (b) and (e), respectively. The electron beam has a Gaussian distribution in both angle $(\Delta \theta=1 \mathrm{mrad})$ and energy $(0.01 \%$ relative FWHM) domain with central energy $\varepsilon=20 \mathrm{~m}$. The pulse length for $\xi_{1}$ is 16000 cycles, while the pulse length for $\xi_{2}$ is the same as the electron beam length, which is about 750 cycles.

can we control the number of harmonics contained in the spectrum? To address them, we turn to analytical estimations. The emission phase is a key variable $\psi=$ $\psi_{n p} t-z_{1} \sin \omega_{1} t-z_{2} \sin \omega_{2} t-z_{3} \sin \Delta \omega_{12} t$, with $\psi_{n p}=$ $\varepsilon u\left(1-v_{z} \cos \theta\right), z_{1}=\left(m \xi_{1} u / \omega_{1}\right) \sin \theta, z_{2}=\left(m \xi_{2} u / \omega_{2}\right) \sin \theta$, $z_{3}=2 \omega_{0} m^{2} \xi_{1} \xi_{2} u /\left(\varepsilon \Delta \omega_{12}^{2}\right), \Delta \omega_{12}=\omega_{1}-\omega_{2}$, and $u=$ $\omega^{\prime} /\left(\varepsilon-\omega^{\prime}\right)$. The integral in Eq. (1) over this phase yields multiplications of Bessel functions with arguments $z_{1}, z_{2}$, $z_{3}[52,53]$. From the phase follows the energy-momentum conservation, determining the emitted photon energy [50]:

$$
\omega^{\prime}=\frac{\omega_{s_{1}, s_{2}}^{m}}{1+2 \gamma_{*}^{2}(1-\cos \theta)},
$$

where $\omega_{s_{1}, s_{2}}^{m}=2 \gamma_{*}^{2}\left(s_{1} \omega_{1}+s_{2} \omega_{2}\right)$ with $s_{1}, s_{2}$ being the numbers of photons absorbed from the first and second pulse, respectively, and $\gamma_{*}=\varepsilon / m_{*}$, with the effective mass $m_{*} \equiv m \sqrt{1+\xi_{1}^{2}+\xi_{2}^{2}}$. The spacing between sequential $s_{2}$ harmonics according to Eq. (2) is $\Delta \omega^{\prime}=2 \gamma_{*}^{2} \omega_{2} \approx \omega_{0}$
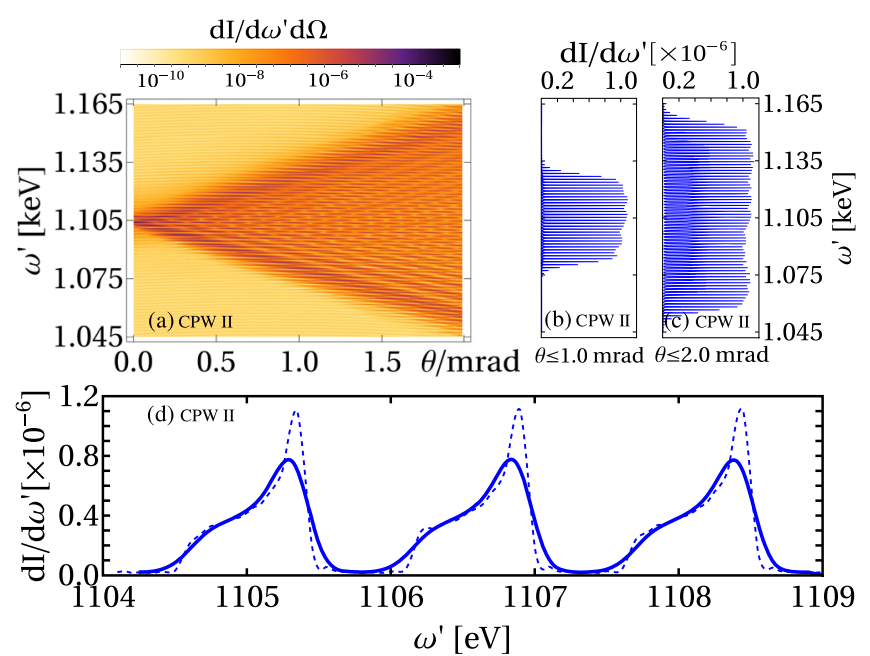

FIG. 3. (a) The angle-resolved spectra for CPW Case II. The angle-integrated spectra for CPW Case II: (b) within $\theta \leq 1 \mathrm{mrad}$; (c) within $\theta \leq 2 \mathrm{mrad}$; (d) spectrum from a realistic electron beam for CPW Case II (solid) and a monochromatic electron beam (dashed). The electron beam has the same distributions as in Fig. 2 but with central energy $\varepsilon=30 \mathrm{~m}$. The pulse length for $\xi_{1}$ is 8000 cycles, while the pulse length for $\xi_{2}$ is the same as the electron beam length, which is about 750 cycles.

because $\omega_{1} / \omega_{2} \approx 4 \gamma_{*}^{2}$ and $\omega_{1}=2 \omega_{0}$. The rather broad emission frequency region in Figs. 2 and 3 follows from Eq. (2). Each line in Figs. 2(d) and 3(a) represents the location of a single harmonic with respect to $\omega_{2}$. The spacing between adjacent lines is $\omega_{0}$.

To obtain a narrow $\mathrm{BW}$ radiation, one needs to apply the emission angle window $\left(\Delta \theta_{w}\right)$. The width of the harmonic due to this finite angle window based on Eq. (2) is

$$
\delta \omega_{w}^{\prime}=\omega_{s_{1}, s_{2}}^{m}\left(\gamma_{*} \Delta \theta_{w}\right)^{2} .
$$

In both cases, $\mathrm{CS}$ and $\mathrm{CPW}$, the most beneficial for a small $\mathrm{BW}$ is the region near forward direction $(\theta=0)$ when $\partial \omega^{\prime} / \partial \theta=0$. Then, at applying a rather small angle window $\Delta \theta_{w}$, the $\mathrm{BW}$ is not $\Delta \theta_{w}$-dependent but determined by the dynamical linewidth. The latter is smaller for CPW in comparison to $\mathrm{CS}$.

The dynamical width of the harmonics is affected by two factors. The first stems from the characteristics of the electron dynamics. From the Bessel function features, the CPW harmonic width is estimated via $z_{2} \approx 1$ [50]. For the main peak,

$$
\delta \omega_{c}^{\prime}=\frac{\omega_{2}}{8}\left(\frac{m_{*}}{m \xi_{2}}\right)^{2} .
$$

The latter BW scaling is surprising. In contrast to CS, the harmonics become narrower for increasing electron energy, confirmed with numerical calculations [50]. For the considered parameters, $\delta \omega_{c}^{\prime} \ll \omega_{0}$, which results in an 
enhancement of satellite peaks over the spectral CS envelope. In fact, adding the second laser $\xi_{2}$ to the CS setup, new satellite peaks arise with a $\omega_{0}$ separation, and the smooth energy distribution within a large BW for CS, $\delta \omega_{C S}^{\prime} \sim \omega_{0} \gamma^{2} / \xi_{1}^{2}$ [50], roams into sharp spikes.

The second contribution to the harmonic width arises from the finite duration of the laser pulses. Note that the duration of the first pulse should be much longer, $N_{1}=n N_{2}$, where $n=\omega_{1} / \omega_{2}$ and $N_{1,2}$ are the numbers of cycles experienced by a single electron during the interaction. Therefore, the photon uncertainty width is mostly determined by the second pulse:

$$
\delta \omega_{f}^{\prime}=2 \gamma_{*}^{2} \omega_{2} / N_{2}=\omega_{0} / N_{2} .
$$

Controlling the angle range $\Delta \theta_{w}$, one can tune $\delta \omega_{w}^{\prime}$ mentioned above such as not to exceed the dynamical width: $\delta \omega_{i n}^{\prime}=\max \left(\delta \omega_{c}^{\prime}, \delta \omega_{f}^{\prime}\right)$ [50]. Please note that the pulse length for $\xi_{2}$ and the electron beam in a realistic experimental setup should be similar such that all the electrons in the beam will contribute to the emitted $\mathrm{x}$-ray pulse during the interaction [50].

In Case II [Fig. 3], the photon energy $\omega^{\prime}$ is less sensitive to the emission angle than in Case I because of a smaller velocity $v_{z}$ at the same energy (at different effective masses), and the number of harmonics is significantly larger than in Case I because $m_{*} / m \xi_{2} \approx \xi_{1} / \xi_{2} \ll 1$. We can assess the effective number of harmonics $\Delta s_{2}$ for a given angle window $\Delta \theta_{w}$ by requiring $z_{2} / \Delta s_{2}=\delta$, with a choice $\delta \approx 0.8$, according to the Bessel function properties [50]:

$$
\Delta s_{2} \approx \frac{1}{\delta}\left(\frac{\Delta \theta_{w}}{\theta_{c}}\right), \quad \theta_{c}=\frac{m_{*}}{8 m \xi_{2}} \frac{1}{\gamma_{*}^{3}},
$$

where $\theta_{c}$ corresponds to the angle when $\delta \omega_{w}^{\prime}\left(\theta_{c}\right)$ is equal to the characteristic width of the harmonics. The integrated spectra over a different angle range in Figs. 3(b),(c) for Case II reveals a trade-off. On the one hand, the range of the comb can be extended by increasing the angle window. On the other hand, it also induces a larger background in the gap between two sequential harmonics [50]. One should balance between the width of a single harmonic and the range of the total comb to have an optimized x-ray comb.

The emittance and energy spreading of the electron beam will cause broadening of the radiation BW and suppress the brilliance. Is it possible with a realistic electron beam in the CPW setup to obtain brilliance better than in CS and generate the comb structure? From Eq. (2) we may estimate the contribution of energy $\Delta \gamma / \gamma$ and angular $\Delta \theta$ spread in the BW broadening as $\delta \omega^{\prime} / \omega^{\prime} \sim \max \left\{\Delta \gamma / \gamma,(\gamma \Delta \theta)^{2}\right\}$. Using electron beam parameters envisaged in [58], $\Delta \gamma / \gamma \sim$ $10^{-4}$ and $\Delta \theta \approx p_{\perp} / p_{z} \sim 10^{-3}$, we can expect the BW broadening up to $\delta \omega^{\prime} / \omega^{\prime} \sim 10^{-4}$. We have tested our conclusion by numerical calculations of the spectra for an electron beam having a Gaussian distribution in both energy and angle domain with relative FWHM equal to $10^{-4}$ and $10^{-3}$, respectively [Figs. 2(g),(h), 3(d)]. Let us discuss the brilliance issue. The brilliance for both CPW and CS cases is the largest in the forward direction [50]. In the case of angle window $\Delta \theta_{w}=0.2 \mathrm{mrad}$ in the forward direction [Figs. 2(b), (e)], the relative BW in CS for a single electron is $\Delta \omega^{\prime} / \omega^{\prime} \approx 10^{-4}$, while in CPW Case I it is 2 times smaller due to smallness of the dynamical BW. However, the number of photons in the line in the CPW case is about 7 times larger than in CS, which is due to more prominent forward emission in CPW. In the case of the electron beam with above parameters, the BW for CS is 1.4 times larger than for CPW, leaving, however, the number of photons in the emission line equal to the case without spreading. Thus, we can have an order of magnitude larger brilliance in the CPW Case I with respect to CS with the same laser and electron parameters. For instance, using electron beam with $10 \mathrm{pC}$ charge and emittance of $0.1 \mathrm{~mm} \cdot \mathrm{mrad}$ (with angle spreading $\Delta \theta=1 \mathrm{mrad}$ and transverse radius of $0.1 \mathrm{~mm}$ ) and $0.01 \%$ energy spreading at electron energy $\varepsilon=10 \mathrm{MeV}$ [58], the peak brilliance in forward direction for the CPW Case $\mathrm{I}$ is $\mathcal{B} \sim 5.2 \times 10^{18} \mathrm{ph} /\left(\mathrm{s} \cdot \mathrm{mrad}^{2} \cdot \mathrm{mm}^{2} \cdot 0.1 \% \mathrm{BW}\right)$, while in $\mathrm{CS}$ it is $\mathcal{B} \sim 6.5 \times 10^{17} \mathrm{ph} /\left(\mathrm{s} \cdot \mathrm{mrad}^{2} \cdot \mathrm{mm}^{2} \cdot 0.1 \% \mathrm{BW}\right)$.

The comblike structure is also preserved when using the electron beam with spreading parameters as above. Thus, Fig. 3(d) shows that in the case of an angular window $\Delta \theta_{w}=2 \mathrm{mrad}$, the electron beam spreading induces a twofold increase of BW. However, the comb structure, with numbers of peaks in the comb $\Delta s_{2}=70$, is still preserved, characterized by the comb visibility, the ratio of the BW to the peak separation, being equal to 0.37. The decrease of the angle window will improve the comb visibility but will decrease the comb length.

Summarizing our approach, first, configuration I or II is chosen depending on the preference of the spectral shape: a single peak or a frequency comb. Then, the desired energy

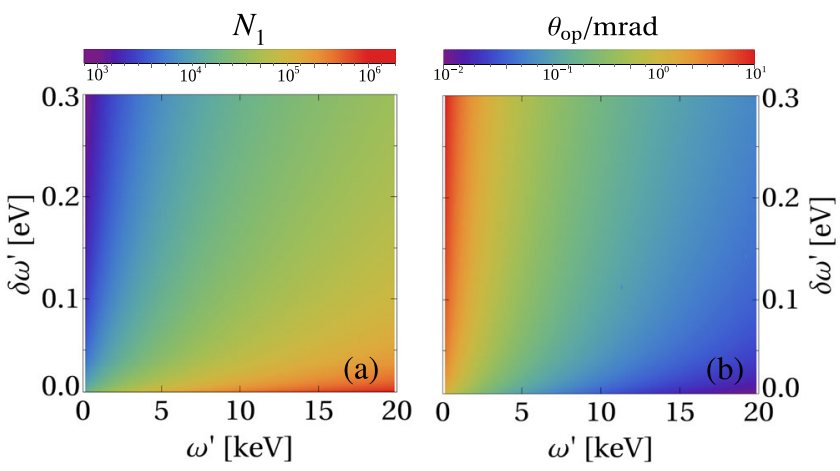

FIG. 4. (a) Required pulse length $N_{1}$ of the $\xi_{1}$ laser for different emitted photon energies $\omega^{\prime}$ with different harmonic peak widths $\delta \omega^{\prime}$ in the spectrum. (b) Optimized angle range $\theta_{o p}$ for different emitted photon energies $\omega^{\prime}$ with different harmonic peak widths $\delta \omega^{\prime}$ when we choose the pulse length in panel (a). 
location and width are specified. The energy $\omega^{\prime}$ determines the effective relativistic factor $\gamma_{*}$ via Eq. (2). Then, from the chosen width $\delta \omega^{\prime}$ and relation, Eq. (3), the angle window $\theta_{w}$ follows. For Case II, where $\delta \omega_{f}^{\prime}$ dominates $\delta \omega_{i n}^{\prime}$, we also require $\delta \omega_{f}^{\prime}=\delta \omega^{\prime}$, from which the duration of the second pulse is evaluated. For a given laser pulse energy and the pulse duration, the field amplitude is derived, which determines the effective mass, and from $\gamma_{*}$ one finds the electron energy (Fig. 4). For a given BW, the number of cycles is determined according to Eq. (5). However, increasing $\omega^{\prime}$ requires a longer duration of the first pulse due to increase of $n=\omega_{1} / \omega_{2} \approx 4 \gamma_{*}^{2}$. The optimal angle window $\theta_{w}$, according to Eq. (2), is narrower at higher $\omega^{\prime}$.

In conclusion, the discussed CPW setup allows one to generate an extremely narrow $\mathrm{BW}$ and collimated $\mathrm{x}$-ray beam in the range of several hundreds of $\mathrm{eV}$ to tens of $\mathrm{keV}$, with brilliance by an order of magnitude exceeding that for CS corresponding to the same laser and electron beam parameters. By tuning the intensity of the two laser pulses, one can produce either a single peak or a comblike $\mathrm{x}$-ray source. This radiation source is attractive for several applications. First, its narrow-band features allow for resonant excitation spectroscopy of highly charged ions [41,42,59-61] without the use of a monochromator as opposed to synchrotron sources. Furthermore, by using the comb, one can probe a much larger energy range in a single shot. Second, the flux and BW of this source render it suitable to operate it as an X-ray free-electron laser seeder, thus replacing the complex and cumbersome self-seeding unit [62-64], which is the only available seeding technique above energies of $100 \mathrm{eV}$. Third, owing to the low angle spread, it is favorable as a source for small angle scattering diagnostics [39]. Fourth, the comblike structure can be employed for extension of plasma diagnostics from the optical and UV range [65-67] to the hard x-ray domain, e.g., for a temporally resolved measurement of the density profile of overdense plasmas. The proposed x-ray comb structure will enable probing much higher density plasma in a large density range in a single shot with high accuracy due to the ultranarrow BW of the single peak in the comb. Finally, replacing the laser fields with strong THz ones, the presented idea can be extended to a hard x-ray frequency comb for ultrahigh precision metrology.

Q.Z.L wishes to thank Zoltán Harman for helpful discussions regarding the applications of the x-ray frequency comb structure. We all thank Jörg Evers for useful comments on this Letter.

*Corresponding author. qingzheng.lyu@mpi-hd.mpg.de ${ }^{\dagger}$ Corresponding author. erez.raicher@mail.huji.ac.il
[1] X-Ray Diffraction: Modern Experimental Technique, edited by B. M. Murphy and O. H. Seeck (Taylor and Francis Group, London, 2014), p. 420.

[2] C. Sanchez-Cano et al., X-ray-based techniques to study the nano-bio interface, ACS Nano 15, 3754 (2021).

[3] I. M. Ternov, Synchrotron radiation, Sov. Phys.-Usp. 38, 409 (1995).

[4] K. Wille, Synchrotron radiation sources, Rep. Prog. Phys. 54, 1005 (1991).

[5] T. Nakazato, M. Oyamada, N. Niimura, S. Urasawa, O. Konno, A. Kagaya, R. Kato, T. Kamiyama, Y. Torizuka, T. Nanba, Y. Kondo, Y. Shibata, K. Ishi, T. Ohsaka, and M. Ikezawa, Observation of Coherent Synchrotron Radiation, Phys. Rev. Lett. 63, 1245 (1989).

[6] H.-P. Schlenvoigt, K. Haupt, A. Debus, F. Budde, O. Jäckel, S. Pfotenhauer, H. Schwoerer, E. Rohwer, J. G. Gallacher, E. Brunetti, R. P. Shanks, S. M. Wiggins, and D. A. Jaroszynski, A compact synchrotron radiation source driven by a laser-plasma wakefield accelerator, Nat. Phys. 4, 130 (2008).

[7] H. N. Chapman, X-ray imaging beyond the limits, Nat. Mater. 8, 299 (2009).

[8] C. Pellegrini, A. Marinelli, and S. Reiche, The physics of X-ray free-electron lasers, Rev. Mod. Phys. 88, 015006 (2016).

[9] C. Bostedt, S. Boutet, D. M. Fritz, Z. Huang, H. J. Lee, H. T. Lemke, A. Robert, W. F. Schlotter, J. J. Turner, and G. J. Williams, Linac coherent light source: The first five years, Rev. Mod. Phys. 88, 015007 (2016).

[10] The European X-Ray Laser Project (XFEL) (2011), https:// www.xfel.eu/.

[11] The Linac Coherent Laser Source (LCLS) (2011), https:// lcls.slac.stanford.edu/.

[12] Z. Zhao, D. Wang, Q. Gu, L. Yin, G. Fang, M. Gu, Y. Leng, Q. Zhou, B. Liu, C. Tang et al., Sxfel: A soft x-ray free electron laser in China, Synchrotron Radiat. News 30, 29 (2017).

[13] A. Ting, R. Fischer, A. Fisher, K. Evans, R. Burris, J. Krall, E. Esarey, and P. Sprangle, Observation of $20 \mathrm{ev}$ x-ray generation in a proof-of-principle laser synchrotron source experiment, J. Appl. Phys. 78, 575 (1995).

[14] R. W. Schoenlein, W. P. Leemans, A. H. Chin, P. Volfbeyn, T. E. Glover, P. Balling, M. Zolotorev, K.-J. Kim, S. Chattopadhyay, and C. V. Shank, Femtosecond x-ray pulses at $0.4 \AA$ generated by 90 thomson scattering: A tool for probing the structural dynamics of materials, Science $\mathbf{2 7 4}$, 236 (1996).

[15] I. Sakai, T. Aoki, K. Dobashi, M. Fukuda, A. Higurashi, T. Hirose, T. Iimura, Y. Kurihara, T. Okugi, T. Omori, J. Urakawa, M. Washio, and K. Yokoya, Production of high brightness gamma-rays through backscattering of laser photons on high-energy electrons, Phys. Rev. ST Accel. Beams 6, 091001 (2003).

[16] D. J. Gibson, S. G. Anderson, C. P. J. Barty, S. M. Betts, R. Booth, W. J. Brown, J. K. Crane, R. R. Cross, D. N. Fittinghoff, F. V. Hartemann, J. Kuba, G. P. Le Sage, D. R. Slaughter, A. M. Tremaine, A. J. Wootton, E. P. Hartouni, P. T. Springer, and J. B. Rosenzweig, Pleiades: A picosecond compton scattering X-ray source for advanced backlighting and time-resolved material studies, Phys. Plasmas 11, 2857 (2004). 
[17] A. Rousse, K. Ta Phuoc, R. Shah, A. Pukhov, E. Lefebvre, V. Malka, S. Kiselev, F. Burgy, Jean-P. Rousseau, D. Umstadter, and D. Hulin, Production of a Kev X-Ray Beam from Synchrotron Radiation in Relativistic LaserPlasma Interaction, Phys. Rev. Lett. 93, 135005 (2004).

[18] S. Corde, K. Ta Phuoc, G. Lambert, R. Fitour, V. Malka, A. Rousse, A. Beck, and E. Lefebvre, Femtosecond x rays from laser-plasma accelerators, Rev. Mod. Phys. 85, 1 (2013).

[19] A. Benedetti, M. Tamburini, and C. H. Keitel, Giant collimated gamma-ray flashes, Nat. Photonics 12, 319 (2018).

[20] A. Sampath et al., Extremely Dense Gamma-Ray Pulses in Electron Beam-Multifoil Collisions, Phys. Rev. Lett. 126, 064801 (2021).

[21] F. V. Hartemann, F. Albert, C. W. Siders, and C. P. J. Barty, Low-Intensity Nonlinear Spectral Effects in Compton Scattering, Phys. Rev. Lett. 105, 130801 (2010).

[22] G. A. Krafft and G. Priebe, Compton sources of electromagnetic radiation, Rev. Accel. Sci. Techol. 03, 147 (2010).

[23] C. Chang, C. Tang, and J. Wu, High-Gain ThompsonScattering X-Ray Free-Electron Laser by Time-Synchronic Laterally Tilted Optical Wave, Phys. Rev. Lett. 110, 064802 (2013).

[24] S. Chen, N. D. Powers, I. Ghebregziabher, C. M. Maharjan, C. Liu, G. Golovin, S. Banerjee, J. Zhang, N. Cunningham, A. Moorti, S. Clarke, S. Pozzi, and D. P. Umstadter, MevEnergy X Rays from Inverse Compton Scattering with Laser-Wakefield Accelerated Electrons, Phys. Rev. Lett. 110, 155003 (2013).

[25] F. V. Hartemann and S. S. Q. Wu, Nonlinear Brightness Optimization in Compton Scattering, Phys. Rev. Lett. 111, 044801 (2013).

[26] G. Sarri, D. J. Corvan, W. Schumaker, J. M. Cole, A. Di Piazza, H. Ahmed, C. Harvey, C. H. Keitel, K. Krushelnick, S. P. D. Mangles, Z. Najmudin, D. Symes, A. G. R. Thomas, M. Yeung, Z. Zhao, and M. Zepf, Ultrahigh Brilliance Multi-Mev Gamma-Ray Beams from Nonlinear Relativistic Thomson Sscattering, Phys. Rev. Lett. 113, 224801 (2014).

[27] K. Khrennikov, J. Wenz, A. Buck, J. Xu, M. Heigoldt, L. Veisz, and S. Karsch, Tunable All-Optical Quasimonochromatic Thomson X-Ray Source in the Nonlinear Regime, Phys. Rev. Lett. 114, 195003 (2015).

[28] I. Ghebregziabher, B. A. Shadwick, and D. Umstadter, Spectral bandwidth reduction of thomson scattered light by pulse chirping, Phys. Rev. ST Accel. Beams 16, 030705 (2013).

[29] B. Terzić, K. Deitrick, A. S. Hofler, and G. A. Krafft, NarrowBand Emission in Thomson Sources Operating in the HighField Regime, Phys. Rev. Lett. 112, 074801 (2014).

[30] D. Seipt, S. G. Rykovanov, A. Surzhykov, and S. Fritzsche, Narrowband inverse compton scattering X-ray sources at high laser intensities, Phys. Rev. A 91, 033402 (2015).

[31] V. Yu Kharin, D. Seipt, and S. G. Rykovanov, HigherDimensional Caustics in Nonlinear Compton Scattering, Phys. Rev. Lett. 120, 044802 (2018).

[32] C. Maroli, V. Petrillo, I. Drebot, L. Serafini, B. Terzić, and G. A. Krafft, Compensation of non-linear bandwidth broadening by laser chirping in thomson sources, J. Appl. Phys. 124, 063105 (2018).

[33] D. Seipt, V. Yu Kharin, and S. G. Rykovanov, Optimizing Laser Pulses for Narrow-Band Inverse Compton Sources in the High-Intensity Regime, Phys. Rev. Lett. 122, 204802 (2019).

[34] M. A. Valialshchikov, V. Yu Kharin, and S. G. Rykovanov, Narrow Bandwidth Gamma Comb from Nonlinear Compton Scattering using the Polarization Gating Technique, Phys. Rev. Lett. 126, 194801 (2021).

[35] A. D. Debus, M. A. Bussmann, M. A. Siebold, A. A. Jochmann, U. A. Schramm, T. E. A. Cowan, and R. A. Sauerbrey, Traveling-wave thomson scattering and optical undulators for high-yield euv and x-ray sources, Appl. Phys. B 100, 61 (2010).

[36] A. Jochmann, A. Irman, M. Bussmann, J. P. Couperus, T. E. Cowan, A. D. Debus, M. Kuntzsch, K. W. D. Ledingham, U. Lehnert, R. Sauerbrey, H. P. Schlenvoigt, D. Seipt, Th. Stöhlker, D. B. Thorn, S. Trotsenko, A. Wagner, and U. Schramm, High Resolution Energy-Angle Correlation Measurement of Hard X Rays from Laser-Thomson Backscattering, Phys. Rev. Lett. 111, 114803 (2013).

[37] F. E. Carroll, M. H. Mendenhall, R. H. Traeger, C. Brau, and J. W. Waters, Pulsed tunable monochromatic x-ray beams from a compact source: New opportunities, Am. J. Roentgenol. 181, 1197 (2003).

[38] D. C. F. Wieland, M. A. Schroer, A. Yu Gruzinov, C. E. Blanchet, C. M. Jeffries, and D. I. Svergun, Asaxs measurements on ferritin and apoferritin at the biosaxs beamline p12 (petra iii, desy), J. Appl. Crystallogr. 54, 830 (2021).

[39] T. Li, A. J. Senesi, and B. Lee, Small angle x-ray scattering for nanoparticle research, Chem. Rev. 116, 11128 (2016).

[40] G. L. Hura, A. L. Menon, M. Hammel, R. P. Rambo, F. L. Poole Ii, S. E. Tsutakawa, F. E. Jenney Jr, S. Classen, K. A. Frankel, R. C. Hopkins et al., Robust, high-throughput solution structural analyses by small angle x-ray scattering (saxs), Nat. Methods 6, 606 (2009).

[41] S. Bernitt, G. V. Brown, J. K. Rudolph, R. Steinbrügge, A. Graf, M. Leutenegger, S. W. Epp, S. Eberle, K. Kubiček, V. Mäckel et al., An unexpectedly low oscillator strength as the origin of the fe xvii emission problem, Nature (London) 492, 225 (2012).

[42] S. Kühn et al., High Resolution Photoexcitation Measurements Exacerbate the Long-Standing fe XVII Oscillator Strength Problem, Phys. Rev. Lett. 124, 225001 (2020).

[43] T. J. Bürvenich, J. Evers, and C. H. Keitel, Nuclear Quantum Optics with X-Ray Laser Pulses, Phys. Rev. Lett. 96, 142501 (2006).

[44] Ralf Röhlsberger, Hans-Christian Wille, Kai Schlage, and Balaram Sahoo, Electromagnetically induced transparency with resonant nuclei in a cavity, Nature (London) 482, 199 (2012).

[45] K. P. Heeg, A. Kaldun, C. Strohm, P. Reiser, C. Ott, R. Subramanian, D. Lentrodt, J. Haber, H.-C. Wille, S. Goerttler, R. Rüffer, C. H. Keitel, R. Röhlsberger, T. Pfeifer, and J. Evers, Spectral narrowing of X-ray pulses for precision spectroscopy with nuclear resonances, Science (N.Y.) 357, 375 (2017).

[46] K. P. Heeg, A. Kaldun, C. Strohm, C. Ott, R. Subramanian, D. Lentrodt, J. Haber, H.-C. Wille, S. Goerttler, R. Rüffer, C. H. Keitel, R. Röhlsberger, T. Pfeifer, and Jörg Evers, Coherent X-ray-optical control of nuclear excitons, Nature (London) 590, 401 (2021).

[47] F. Vagizov, V. Antonov, Y. V. Radeonychev, R. N. Shakhmuratov, and O. Kocharovskaya, Coherent control 
of the waveforms of recoilless $\gamma$-ray photons, Nature (London) 508, 80 (2014).

[48] A. I. Chumakov, A. Q. R. Baron, I. Sergueev, C. Strohm, O. Leupold, Y. Shvyd'ko, G. V. Smirnov, R. Rüffer, Y. Inubushi, M. Yabashi et al., Superradiance of an ensemble of nuclei excited by a free electron laser, Nat. Phys. 14, 261 (2018).

[49] E. Kuznetsova and O. Kocharovskaya, Quantum optics with X-rays, Nat. Photonics 11, 685 (2017).

[50] See Supplemental Material at http://link.aps.org/ supplemental/10.1103/PhysRevLett.128.024801 for more details of the derivations as well as additional explanations and figures.

[51] V. N. Baier, V. M. Katkov, and V. M. Strakhovenko, Electromagnetic Processes at High Energies in Oriented Single Crystals (World Scientific, Singapore, 1994).

[52] Q. Z. Lv, E. Raicher, C. H. Keitel, and K. Z. Hatsagortsyan, Anomalous violation of the local constant field approximation in colliding laser beams, Phys. Rev. Research 3, 013214 (2021).

[53] Q. Z. Lv, E. Raicher, C. H. Keitel, and K. Z. Hatsagortsyan, Ultrarelativistic electrons in counterpropagating laser beams, New J. Phys. 23, 065005 (2021).

[54] C. Gohle, T. Udem, M. Herrmann, J. Rauschenberger, R. Holzwarth, H. A. Schuessler, F. Krausz, and T. W. Hänsch, A frequency comb in the extreme ultraviolet, Nature (London) 436, 234 (2005).

[55] A. Cingöz, D. C. Yost, T. K. Allison, A. Ruehl, M.E. Fermann, I. Hartl, and J. Ye, Direct frequency comb spectroscopy in the extreme ultraviolet, Nature (London) 482, 68 (2012).

[56] S. M. Cavaletto, Z. Harman, C. Ott, C. Buth, T. Pfeifer, and C. H. Keitel, Broadband high-resolution X-ray frequency combs, Nat. Photonics 8, 520 (2014).

[57] G. Porat, C. M. Heyl, S. B. Schoun, C. Benko, N. Dörre, K. L. Corwin, and J. Ye, Phase-matched extreme-ultraviolet frequency-comb generation, Nat. Photonics 12, 387 (2018).

[58] K. E. Deitrick, G. A. Krafft, B. Terzić, and J. R. Delayen, High-brilliance, high-flux compact inverse compton light source, Phys. Rev. Accel. Beams 21, 080703 (2018).
[59] J. K. Rudolph, S. Bernitt, S. W. Epp, R. Steinbrügge, C. Beilmann, G. V. Brown, Sita Eberle, A. Graf, Zoltan Harman, N. Hell, M. Leutenegger, A. Muller, K. Schlage, H. C. Wille, H. Yavas, J. Ullrich, and J. R. CrespoLopezUrrutia, X-Ray Resonant Photoexcitation: Linewidths and Energies of $K \alpha$ Transitions in Highly Charged Fe Ions, Phys. Rev. Lett. 111, 103002 (2013).

[60] C. Lyu, S. M. Cavaletto, C. H. Keitel, and Z. Harman, Interrogating the Temporal Coherence of EUV Frequency Combs with Highly Charged Ions, Phys. Rev. Lett. 125, 093201 (2020).

[61] J. Nauta, J.-H. Oelmann, A. Borodin, A. Ackermann, P. Knauer, I. S. Muhammad, R. Pappenberger, T. Pfeifer, and J. R. Crespo López-Urrutia, Xuv frequency comb production with an astigmatism-compensated enhancement cavity, Opt. Express 29, 2624 (2021).

[62] I. Nam, C.-K. Min, B. Oh, G. Kim, D. Na, Y. J. Suh, H. Yang, M. H. Cho, C. Kim, M.-J. Kim et al., High-brightness self-seeded x-ray free-electron laser covering the $3.5 \mathrm{kev}$ to 14.6 kev range, Nat. Photonics 15, 435 (2021).

[63] I. Inoue, T. Osaka, T. Hara, T. Tanaka, T. Inagaki, T. Fukui, S. Goto, Y. Inubushi, H. Kimura, R. Kinjo et al., Generation of narrow-band $\mathrm{x}$-ray free-electron laser via reflection selfseeding, Nat. Photonics 13, 319 (2019).

[64] E. Allaria, D. Castronovo, P. Cinquegrana, P. Craievich, M. Dal Forno, M. B. Danailov, G. D’Auria, A. Demidovich, G. De Ninno, S. Di Mitri et al., Two-stage seeded soft-x-ray free-electron laser, Nat. Photonics 7, 913 (2013).

[65] S. Dobosz, G. Doumy, H. Stabile, P. D’Oliveira, P. Monot, F. Réau, S. Hüller, and P. Martin, Probing Hot and Dense Laser-Induced Plasmas with Ultrafast XUV Pulses, Phys. Rev. Lett. 95, 025001 (2005).

[66] P. Gibbon, D. Altenbernd, U. Teubner, E. Förster, P. Audebert, J.-P. Geindre, J.-C. Gauthier, and A. Mysyrowicz, Plasma density determination by transmission of lasergenerated surface harmonics, Phys. Rev. E 55, R6352 (1997).

[67] W. Theobald, R. Hässner, C. Wülker, and R. Sauerbrey, Temporally Resolved Measurement of Electron Densities (>10 23 cm- 3) with High Harmonics, Phys. Rev. Lett. 77, 298 (1996). 